\title{
On the Variations in Capacity of MIMO CommunicationSystems to Channel Perturbations
}

\author{
M.R. Bhavani Shankar, K.V. S.Hari \\ Department of Electrical Communication \\ Engineering, \\ Indian Institute of Science, Bangalore \\ bshankareprotocol.ece.iisc. ernet. in \\ hari@ece.iisc.ernet. in
}

\begin{abstract}
It is known that the capacity of a multi-antenna flat non-fading gaussian channels (MIMO capacity) can be improved if the transmitter has perfect knowledge of channel. The channel information affects the input allocation and hence there is capacity degradation when the transmitter does not know the channel perfectly. In this work variation in MIMO capacity due to small perturbations that do not change the rank of the channel is studied. The need for the analysis is highlighted by the fact that at high SNR, most channel estimation algorithms give small errors. Expressions and bounds for capacity are derived using first order perturbation analysis. Further to this, the dependence of capacity on the channel parameters and total power is studied. It is shown that the upper bound on degradation in capacity for small errors varies as the square of the spectral norm of perturbation. Further the degradation has an approximate inverse square relation with total power used and channel singular values. These results are extended to a general class of communication systems and results for MIMOOFDM systems over frequency selective channels are presented.
\end{abstract}

\section{INTRODUCTION}

Consider the MIMO (Multiple Input Multiple Output) system with $\mathrm{m}$ transmitting and $\boldsymbol{n}$ receiving antennas over Gaussian channel. Specifically, we are interested in the model,

$$
\not{y}=\mathrm{H} \underline{x}+\underline{z}
$$

where $\forall, \underline{x}$ are $\mathrm{n} \times 1, \mathrm{~m} \times 1$ received and transmitted vectors respectively. $\underline{\mathbf{z}}$ is $\mathbf{n} \mathbf{x} \mathbf{1}$ complex Gaussian vector with independent identically distributed real and imaginary parts with zero mean and variance 0.5 [1]. The channel, denoted by the $\mathrm{n} \times \mathrm{m}$ matrix $\mathrm{H}$, is assumed to be complex, flat and non-fading. Extensions to frequency selective non-fading channel is provided later. $\mathrm{Q}$ denotes the input correlation matrix of the signals transmitted across antennas. The power available for transmission is $\mathbf{P}$, so that $\operatorname{Trace}(\mathbf{Q}) \leq \mathrm{P}$. The capacity for such a setup is [1]

$$
\begin{aligned}
C_{\text {opt }} & \left.=\sum_{i=1}^{\min (m, n)} \ln \left(\mu \lambda_{i}(\mathbf{A})\right)\right)^{+} \\
\text {where } P & =\sum_{i}\left(\mu-\lambda_{i}^{-1}(\mathbf{A})\right)^{+}, \mathbf{A}=\mathbf{H}^{*} \mathbf{H}
\end{aligned}
$$

where $(a)^{+}=\max \{0, a\}$ and ${ }^{*}$ denotes Hermitian operation. $\lambda_{i}(\mathbf{Y})$ and $\mathbf{Y}_{(i, j)}$, respectively, are the $i^{t h}$ eigen-value and the $(i, j)^{\text {th }}$ entry of a matrix $\boldsymbol{Y}$.Further $\left\{\lambda_{i}(\mathbf{A})\right\}$ are arranged in descending order.

If the transmitter has perfect channel information, then it obtains the optimal input matrix $\mathbf{Q}_{o p t}$ and transmits accordingly so that capacity $C_{\text {opt }}$ is achieved. However, if the transmitter has an estimate of the channel given by $[\mathrm{H}+\mathrm{AH}]$, the matrix $\widehat{\mathrm{Q}}_{\text {opt }}$. optimal for $[\mathrm{H}+\mathrm{AH}]$ is obtained and transmission carried out accordingly over actual channel $\mathbf{H}$. Such a situation, for example, arises when the receiver measures a channel with error and relays the same information to the transmitter for input optimization. While the transmitter assumes that the mutual information achieved is $\widehat{C}_{\text {opt }}$ (the capacity for the perturbed channel), the actual mutual information achieved is $\mathrm{C}$. Since $C_{\text {opt }}$ is optimal for $\mathrm{H}, C \leq C_{\text {opt }}$. We term $C$ as achieved capacity. The expressions for $\widehat{C}_{\text {opt }}, C_{\text {opt }}$ and $C$ are [1]

$$
\begin{gathered}
\widehat{C}_{o p l}=\log \operatorname{det}\left[\mathbf{I}_{n}+[\mathrm{H}+\Delta \mathbf{H}] \widehat{\mathbf{Q}}_{o p t}[\mathbf{H}+\mathrm{AH})^{\prime}\right] \\
C_{o p t}=\log \operatorname{det}\left[\mathbf{I}_{n}+\mathrm{HQ}_{o p t} \mathrm{H}^{*}\right] \\
O=\log \operatorname{det}\left[\mathrm{I}_{n}+\mathrm{H} \widehat{\mathbf{Q}}_{o p t} \mathrm{H}^{*}\right]
\end{gathered}
$$

where the logarithm is over the base $\mathbf{2}$ throughout the paper. Since we are dealing with fixed channels, $C$ is independent of receiver channel information.

We are interested in the variation of $C$ with channel perturbation inorder to evaluate the necessity of precise channel information and algorithms that yield such information. While one may numerically evaluate $C$ from equation 3 for a given $\mathrm{H}$ and $\mathrm{AH}$, doing so will not highlight the effects of various parameters like user power $P$ and channel singular values on $\mathrm{C}$. Further, having an explicit relationship between the aforesaid parameters and $C$ provides the designer with tools to combat the degradation. As a result, we need to have expression for $\mathbf{C}$, more refined than equation 3 , in terms of channel perturbation, channel singular values and total power $\mathbf{P}$.

We use $A+A A=[H+\triangle H]^{*}[H+A H]$ so that $A A$ is the penurbation in A. The spectral norm [2] of a matrix $\boldsymbol{Y}$.denoted by $\|\mathbf{Y}\|$, is used as a "measure" of matrix quantities. It is shown in the paper that, for any full rank channel, the rank of the measured channel is same as that of the true channel if $\|\Delta \mathbf{A}\|$ is less than the minimum eigen-value of $\mathrm{A}$. In this paper, we restrict ourselves to such a class of perturbations which do not change the rank. The need to obtain $C$ for such a class is motivated by the observation that errors produced by most channel estimation algorithms decrease with SNR and for well-conditioned channels the errors belong to aforesaid class. The results for perturbations that change the rank are presented in [4].

The results obtained can be easily extended to those communication systems whose capacity expressions are similar to equation 3, for e.g. MIMO-OFDM (Orthogonal Frequency Division Multi- 
plexing) system [5]. While earlier results are for flat channels, we present results for MIMO-OFDM system over frequency selective channels.

We assume that $\mathrm{A}$ is full rank with distinct eigen-values. This entails that $n \geq m$, i.e, the number of transmit antennas are more than the number of receive antennas.' The reason for this is mentioned in Section II. We do not consider the loss in capacity due to the sharing/ estimation of channel parameters.

\section{PERTURBATION ANALYSIS}

We take recourse to the first order perturbation methods [3] For the sake of completeness we mention the following useful results. Let $\boldsymbol{Y}$ be a $\mathrm{n} \times \mathrm{n}$ Hermitian matrix having distinct eigen values and $\lambda_{i}(\mathrm{Y}), \underline{v}_{i}, 1 \leq i \leq \mathrm{n}$ being the eigen-values and corresponding eigen-vectors respectively . Let the perturbed matrix be $\mathrm{Y}+\mathrm{A} Y$. Then the first order perturbation of $\lambda_{i}(\mathrm{Y})$, and $\underline{v}_{i}$ denoted by $\Delta \lambda_{i}(\mathrm{X})$ and $\Delta \underline{v}_{i}$ respectively, are given by [3]

$$
\begin{aligned}
\Delta \underline{v}_{i} & =\sum_{j=1}^{n} \frac{\underline{v}_{j} \underline{v}_{j}^{*}[\Delta \mathbf{Y}] \underline{v}_{i}}{\lambda_{i}(\mathbf{Y})-\lambda_{j}(\mathbf{Y})} \\
\Delta \lambda_{i}(\mathbf{Y}) & =\underline{v}_{i}^{*}[\Delta \mathbf{Y}] \underline{v}_{i}
\end{aligned}
$$

Denote $\mathrm{X}=\mathrm{I},+\mathrm{HQ}_{\mathrm{op} \iota} \mathrm{H}^{*}$ and $\mathrm{X}+\mathrm{AX}=\mathrm{I},+\mathbf{H} \widehat{\mathbf{Q}}_{\mathrm{opt}} \mathbf{H}^{*}$. Since we restrict ourselves to perturbations that do not change the rarik and to a channel having full rank, we see that rank $\left(\mathbf{H} \widehat{Q}_{o p l} \mathrm{H}^{*}\right)=\operatorname{rank}\left(\mathbf{H Q}_{o p t} \mathbf{H}^{*}\right)=\mathrm{m}$. Arranging the eigenvalues of $\mathrm{X}$ and $\mathrm{X}+\mathrm{AX}$ in descending order, we see that $\lambda_{i}(\mathrm{X})=\lambda_{i}(\mathrm{X}+\mathrm{AX})=1,(m+1) \leq i \leq \mathrm{n}$. Using this and equation 3 , we have, $C_{\text {opt }}=\sum_{i=1}^{m} \log \lambda_{i}(\mathrm{X})$ and $C=$ $\sum_{i=1}^{m} \log \lambda_{i}(\mathrm{X}+\mathrm{AX})$. Hence it suffices to study the perturbation in the first $m$ eigen-values of $X$.

Let the SVD [2] of $\mathrm{H}=\mathrm{UDV}^{*}$, with $\mathbf{U}=\left[\underline{u}_{1}, \underline{u}_{2} \ldots \underline{u}_{n}\right]$ and $\mathrm{V}=\left[\underline{v}_{1}, \underline{v}_{2} \ldots \underline{v}_{n}\right]$ where $\underline{u}_{i}$ and $\underline{v}_{i}$ are $n \times 1$ and $\mathrm{m} \times 1$ column vectors respectively. Then $\mathrm{Q}_{o p t}=\mathrm{VZV}^{*}$ is the EVD [2] of $\mathrm{Q}_{o p t}$ [I] leading to $\mathrm{X}=\mathrm{U}\left(\mathrm{I}_{n}+\mathrm{DZD} \mathrm{D}^{*}\right] \mathrm{U}^{*}$. We assume that iterative water-filling [1] is not used so that $\mathbf{Z}_{(i, i)}=\lambda_{i}\left(\mathbf{Q}_{o p t}\right)$ has closed form solution given by [1],

$$
\lambda_{i}\left(\mathbf{Q}_{o p t}\right)=\frac{-1}{\lambda_{i}(\mathbf{A})}+\frac{1}{m}\left[P+\sum_{j=1}^{m} \frac{1}{\lambda_{i}(\mathbf{A})}\right]
$$

We comment on the choice of $\mathbf{P}$ that yields equation 6 later.

Similarly, EVD of $\widehat{\mathbf{Q}}_{\text {opt }}=[\mathrm{V}+\Delta \mathrm{V}][\mathrm{Z}+\Delta \mathrm{Z}\}[\mathrm{V}+\Delta \mathrm{V})^{*}$ where $[\mathrm{V}+\mathrm{AV}]$ is the eigen-vector matrix of $[\mathrm{A}+\Delta \mathbf{A}]$. Then, $\mathrm{AX}=\mathbf{H}\left[\widehat{\mathbf{Q}}_{\text {opt }}-\mathrm{Q}_{\text {opt }}\right] \mathrm{H}^{*}$, can be written as,

$$
\begin{aligned}
& A X=H\left(V Z[\Delta V]^{*}+(\Delta V) Z V^{*}+V[\Delta Z] V^{*}+V \Delta Z \cdot(7)\right. \\
& \left.[\Delta V]^{*}+[\Delta V] \Delta Z V^{*}+\Delta V(Z) \Delta V^{*}+\Delta V(\Delta Z] \Delta V^{*}\right) H^{*}
\end{aligned}
$$

Neglecting the second order terms, we have from equation 7

$$
\begin{aligned}
\mathrm{AX} & =\mathrm{H}\left[\mathrm{VZ}[\Delta \mathrm{V}]^{*}+[\Delta \mathrm{V}] \mathrm{ZV}^{*}+\mathrm{V}[\Delta \mathrm{Z}] \mathrm{V}^{*}\right] \\
& =\mathrm{UD}\left[\mathrm{Z}[\Delta \mathrm{V}]^{*} \mathrm{~V}+\mathrm{V}^{*}[\Delta \mathrm{V}] \mathrm{Z}+[\Delta \mathrm{Z}\}\right] \mathrm{D}^{*} \mathrm{U}^{*}
\end{aligned}
$$

Using $\lambda_{i}(\mathbf{X})=1+\lambda_{i}(\mathbf{A}) \lambda_{i}\left(\mathbf{Q}_{\text {opt }}\right), 1 \leq i \leq \mathrm{m}$ it is clear that the first $\mathrm{m}$ eigen-values of $\mathrm{X}$ are distinct if $\mathrm{A}$ has distinct eigen-values. Since equation $\mathbf{5}$ is valid for simple eigen-values, we assume that $\mathrm{A}$ and hence first $\mathrm{m}$ eigen-values of $\mathrm{X}$ are simple (distinct). Using equation 5 ,

$$
\begin{aligned}
& \Delta \lambda_{i}(\mathbf{X})=\underline{u}_{i}^{*}\left(\mathrm{UD}\left[\mathbf{Z}[\Delta \mathbf{V}]^{*} \mathbf{V}+\mathrm{V}^{*}[\Delta \mathrm{V} \mid \mathrm{Z}+\Delta \mathrm{Z}] \mathrm{D}^{*} \mathrm{U}^{*}\right) \underline{u}_{i}\right. \\
& =\mathbf{D}_{(i, i)}^{2}\left(\Delta \mathbf{Z}_{(i, i)}+2 \mathbf{Z}_{(i, i)} \operatorname{Real}\left[\mathrm{V}^{*} \Delta \mathrm{V}\right]_{(i, i)}\right), 1 \leq i \leq \mathrm{m}(9)
\end{aligned}
$$

as $\mathrm{Z}$ is a real diagonal matrix.

\section{A. Computing $\Delta \lambda_{i}(\mathbf{X})$}

Note that $\mathbf{V}$ and $\mathrm{V}+\mathrm{AV}$ are the eigen-vector matrices of $\mathrm{A}$ and $\mathrm{A}+\Delta \mathrm{A}$ respectively. Since $\Delta \underline{v}_{i}$ is the perturbation of $\underline{v}_{i}$, from the first order perturbation of equation $\mathbf{4}$ and noting that $\left[\mathbf{V}^{*} \Delta \mathbf{V}\right]_{(i, i)}=\underline{v}_{i}^{*}\left[\triangle \underline{v}_{i}\right]$ we have,

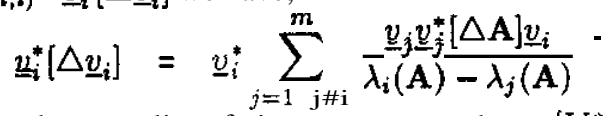

By the orthonormality of eigen-vectors we have, $\left[V^{*} \Delta \mathbf{V}\right]_{(i . i)}$ $=\underline{v}_{i}^{*} \Delta \underline{v}_{i}=0$. From equations 9 and 10 , we have,

$$
\Delta \lambda_{i}(\mathbf{X})=\mathbf{D}_{(i, i)}^{2}\left[\Delta \mathbf{Z}_{(i, i)}\right]=\lambda_{i}(\mathbf{A})\left[\Delta \mathbf{Z}_{(i, i)}\right]
$$

where equations 10 and 9 require $A$ to possess distinct eigenvalues. In finding rank of $\mathbf{H} \widehat{\mathbf{Q}}_{\text {opt }} \mathbf{H}^{*}$ and Appendix $\mathrm{A}, \mathrm{A}$ is required to be full rank. Combining the two, $\mathrm{A}$ is assumed to be full rank with distinct eigen-values.

The perturbation in equation 11 involves the difference of eigen-values of $\mathbf{Q}_{\text {opt }}$ and $\mathbf{Q}_{\text {opt }}$ which are functions of $\mathbf{H}$ and $\mathrm{H}+\mathrm{AH}$. To proceed further, one needs closed form expression for $[\Delta \mathbf{Z}]_{(i, i)}$ in terms of singular values of $\mathbf{H}$ and $\mathbf{H}+\mathrm{AH}$. In the absence of iterative water filling [1], $\lambda_{i}\left(\mathbf{Q}_{\text {opt }}\right)$ is given by equation 6 and $\lambda_{i}\left(\widehat{Q}_{o p t}\right)$ is given by,

$$
\lambda_{i}\left(\widehat{\mathbf{Q}}_{\text {opt }}\right)=\frac{-1}{\lambda_{i}(\mathbf{A}+\Delta \mathbf{A})}+\frac{1}{m}\left[P+\sum_{j=1}^{m} \frac{1}{\lambda_{i}(\mathbf{A}+\Delta \mathbf{A})}\right]
$$

and hence closed form expression for $[\Delta \mathbf{Z}]_{(i, i)}$ exists. However, there exists no closed form expressions for $\lambda_{i}\left(\mathbf{Q}_{\text {opt }}\right), \lambda_{i}\left(\widehat{\mathbf{Q}}_{\text {opt }}\right)$ and $[\Delta \mathbf{Z}]_{(\boldsymbol{i}, \boldsymbol{i})}$, when iterative water filling algorithm is used. Hence, $\mathbf{P}$ is chosen such that $\lambda_{i}\left(\mathbf{Q}_{\text {opt }}\right)$ and $\lambda_{i}\left(\widehat{\mathbf{Q}}_{\text {opt }}\right)$ are as given in 6 and 12. This is achieved by obtaining $\mathbf{P}$ as in Appendix $A$ for a given upper bound on $\|\triangle \mathbf{A}\|$.

Using the first order perturbation for eigen-value?, of $[\mathrm{A}+\mathrm{AA}], \lambda_{i}(\mathbf{A}+\mathrm{AA})=\lambda_{i}(\mathbf{A})+\underline{v}_{i}^{*}(\Delta \mathbf{A}) \underline{v}_{i}, 1 \leq i \leq \mathrm{m}$. We can rewrite this as $\lambda_{i}(\mathbf{A}+\Delta \mathbf{A})=\underline{v}_{i}^{*}(\mathbf{A}+\Delta \mathbf{A}) \underline{v}_{i}$. Since $\mathrm{A}+\mathrm{AA}$ is positive semi-definite, the first order approximation gives a positive (and valid) estimate for $\lambda_{i}(\mathbf{A}+\mathbf{A A})$. Using this in equation $\mathbf{1 2}$, we have,

$$
\lambda_{i}\left(\widehat{Q}_{o p t}\right)=\frac{-1}{\lambda_{i}(\mathrm{~A})+\alpha_{i}}+\frac{1}{m}\left[P+\sum_{j=1}^{m} \frac{1}{\lambda_{j}(\mathrm{~A})+\alpha_{i}}\right]
$$

where $\alpha_{i}=\underline{v}_{i}^{*}[\Delta \mathbf{A}] \underline{v}_{i}, 1 \leq i \leq \mathrm{m}$. Then using $[\Delta \mathbf{Z}]_{(i, i)}=$ $\lambda_{i}\left(\widehat{\mathbf{Q}}_{\text {opt }}\right)-\lambda_{i}\left(\mathbf{Q}_{\text {opt }}\right)$, equations 6,13 and $\mu_{j}=\lambda_{j}(\mathbf{A})+\alpha_{j}, 1 \leq$ $j \leq m$,

$$
[\Delta \mathrm{Z}]_{(i, i)}=\frac{\alpha_{i}}{\lambda_{i}(\mathrm{~A}) \mu_{i}}-\frac{1}{m} \sum_{j=1}^{m} \frac{\alpha_{j}}{\lambda_{j}(\mathrm{~A}) \mu_{j}}, 1 \leq i \leq m
$$

It can be easily shown that $\lambda_{i}(\mathbf{X})=1+\lambda_{i}(\mathbf{A}) \mathbf{Z}_{(i, i)}, 1 \leq i \leq \mathrm{m}$ and $\lambda_{i}(\mathrm{X})=1,(\mathrm{~m}+1) \leq i \leq \mathrm{n}$. Using $\lambda_{i}(\mathbf{X}+\Delta \mathrm{X})=$ $\lambda_{i}(\mathbf{X})+\Delta \lambda_{i}(\mathbf{X})$, and equation $1 \bar{l}$, we have $\lambda_{i}(\mathbf{X}+\mathrm{AX})=1+$ 
$\lambda_{i}(\mathbf{A})[\mathrm{Z}+\Delta \mathrm{Z}]_{(i, i)}, \mathbf{1} \leq i \leq m$ and $\lambda_{i}(\mathrm{X}+\mathrm{AX})=1,(m+$ 1) $\leq i \leq n$.

Using equation 13 and noting that $\mathbf{Z}_{(i, i)}=\lambda_{i}\left(\mathbf{Q}_{\text {opt }}\right)$, we have

$$
\lambda_{i}(\mathrm{X}+\mathrm{AX})=\frac{\alpha_{i}}{\mu_{i}}+\frac{\lambda_{i}(\mathbf{A})}{m}\left[P+\sum_{j=1}^{m} \frac{1}{\mu_{j}}\right], 1 \leq i \leq m
$$

where a; and $\mu_{i}$ are mentioned above.

Using equation 15 and noting that $C=\log \operatorname{det}[\mathbf{X}+\mathbf{A X}]=$ $\sum_{i=1}^{m} \log \lambda_{i}(X+A X)$, we proceed to obtain ihe first order approximation $C_{\text {pert }}$ for $C$. Noting that $\mathbf{a}_{i}=\underline{v}_{i}^{*}[\Delta \mathbf{A}] \underline{v}_{i}=$ $2 \mathbf{D}_{(i, i)} \operatorname{Real}\left(\underline{u}_{i}^{*}[\Delta \mathbf{H}] \underline{v}_{i}\right)+\left\|[\triangle \mathbf{H}] \underline{v}_{i}\right\|_{2}^{2}$ where $\|\bullet\|_{2}$ is the $l_{2}$ norm [2], $C_{\text {pert }}$ is obtained as,

$$
\begin{aligned}
& C_{\text {pert }}=\sum_{i=1}^{m} \log \left(\frac{\alpha_{i}}{\mu_{i}}+\frac{\lambda_{i}(\mathbf{A})}{m}\left[P+\sum_{i=1}^{m}{ }_{\mu_{j}}^{1_{j}}\right]\right) \text { with } \\
& \alpha_{i}=\underline{v}_{i}^{*}[\Delta \mathbf{A}] \underline{v}_{i}, \mu_{i}=\left(\lambda_{i}(\mathbf{A})+\alpha_{i}\right), 1 \leq i \leq m
\end{aligned}
$$

B. Bounds on the degradation in Capacity $C_{\text {opt }}-C_{\text {pert }}$

We are interested in the degradation $C_{\text {opt }}-\mathrm{C}$. Since we assume (and later show) that $C_{\text {pert }}$ matches closely with $C$, we shall deal with $C_{\text {opt }}-C_{\text {pert }}$. Since $C_{\text {opt }}-C_{\text {pert }}=$ $-\sum_{i=1}^{m} \log \left(\frac{\lambda_{i}(\mathbf{X}+\Delta \mathbf{X})}{\lambda_{i}(\mathbf{X})}\right)$. Using $\lambda_{i}(\mathbf{X}+\Delta \mathbf{X})=\lambda_{i}(\mathbf{X})+$ $\Delta \lambda_{i}(\mathbf{X})$, we have, $C_{\text {opt }}-C_{\text {pert }}=-\sum_{i=1}^{m} \log \left(1+\frac{\Delta \lambda_{i}(\mathbf{X})}{\lambda_{i}(\mathbf{X})}\right)$. By Taylor's series expansion,

$$
C_{o p t}^{-}-C_{p e r t}=-\sum_{i=1}^{m}\left[\left(\frac{\Delta \lambda_{i}(\mathbf{X})}{\ln 2 \lambda_{i}(\mathbf{X})}\right)-\frac{0.5}{\ln 2}\left(\frac{\Delta \lambda_{i}(\mathbf{X})}{\lambda_{i}(\mathbf{X})}\right)^{2}\right]
$$

Now, using $\lambda_{i}(\mathbf{X})=.1+\lambda_{i}(\mathbf{A}) \lambda_{i}\left(\mathbf{Q}_{\text {opt }}\right), \quad \mathbf{1} \leq i \leq m$ and equations 11 , 6 we have $\frac{-\Delta \lambda_{i}(\mathbf{X})}{\lambda_{i}(\mathbf{X})}=\frac{m \Delta \mathbf{Z}_{(i, i)}}{P+\gamma}$ where $\gamma=$ $\sum_{i=1}^{m} \frac{1}{\lambda_{i}(\mathbf{A})}$. Due to the power constraint; we have, $\operatorname{Trace}(\mathbf{Z})$ $=\operatorname{Trace}(\mathbf{Z}+\triangle \mathbf{Z})=P$. Thus $\sum_{i=1}^{m}[\Delta \mathbf{Z}]_{(\boldsymbol{i}, i)}=\mathbf{0}$. Using the above form for $\frac{\Delta \lambda_{i}(\mathbf{X})}{\lambda_{\mathbf{z}}(\mathbf{X})}$ and $\sum_{i=1}^{m}[\Delta \mathbf{Z}]_{(i, i)}=0$ in equation 17 ,

$$
C_{\text {opt }}-C_{\text {pert }}=\frac{1}{2 \ln 2} \cdot\left(\frac{m}{P+\gamma}\right)^{2} \sum_{i=1}^{m}\left(\Delta \mathbf{Z}_{(i, i)}\right)^{2}
$$

Using equation 14 in 18 ,

$$
\begin{gathered}
C_{o p t}-C_{\text {pert }}=\frac{1}{2 \ln 2}\left(\frac{m}{P+\gamma}\right)^{2} \sigma \text { where } \\
\sigma=\sum_{i=1}^{m} \beta_{i}^{2}-\frac{1}{m}\left(\sum_{i=1}^{m} \beta_{i}\right)^{2}, \beta_{i}=\frac{\alpha_{i}}{\lambda_{i}(\mathbf{A})\left(\lambda_{i}(\mathbf{A})+\alpha_{i}\right)}
\end{gathered}
$$

Equation 19requires information about the matrix AA. However, using $-\|\Delta \mathbf{A}\| \leq \alpha_{i} \leq\|\Delta \mathbf{A}\|, \forall i$, wegetanupperboundonthe degradation as,

$$
\begin{gathered}
C_{o p t}-C_{p e r t}<\frac{1}{2 \ln 2}\left(\frac{m\|\Delta \mathbf{A}\|}{P+\gamma}\right)^{2} \\
\sum_{i=1}^{m}\left[\frac{1}{\lambda_{i}(\mathbf{A})\left(\lambda_{i}(\mathbf{A})-\|\Delta \mathbf{A}\|\right)}\right]^{2}
\end{gathered}
$$

Consider figure 1, which plots the $C_{\text {pert }}$ and $C$ (obtained explicitly by evaluating equation 3 ) as well as their absolute difference $\left|C_{\text {pert }}-C\right|$ for a full rank 4 x 3 channel of unit norm with random perturbations whose maximum norm is limited to be less than $0.1 \lambda_{3}(\mathbf{A})$. While $\mathbf{P}$ can be used as in Appendix $\mathbf{A}$ for each perturbation, for fair comparison, we fix $\mathrm{P}=\frac{1.1 \mathrm{~m}}{0.9 \lambda_{m}(\mathrm{~A})}$, a value
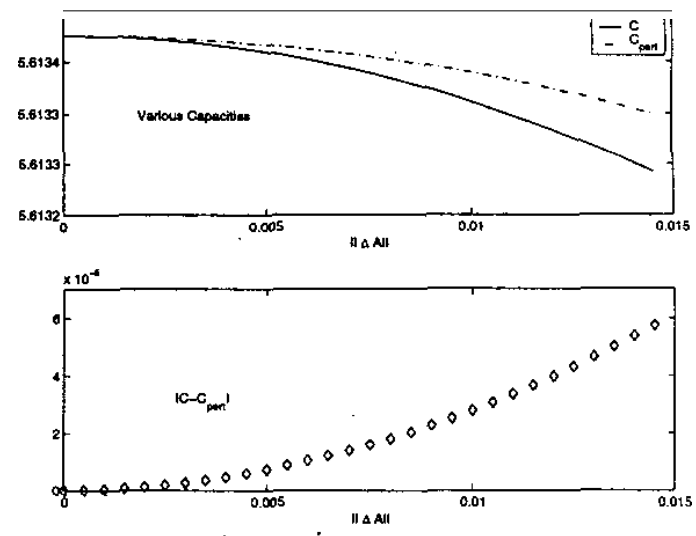

Fig. 1. Perturbation for a channel of norm $1, m=3, n=4: \lambda_{m}(A)=0.2057$. maximum $\|\triangle \mathbf{A}\|=0.015, C_{o p t}=5.6134$.

that meet our requirements for the range of perturbations used ' From this plot and various simulations, we see that $C_{\text {pert }}$ is a good approximation of $C$ for small perturbations. Hence our assumption of studying $C_{o p t}-C_{p e r t}$ instead of $C_{\text {opt }}-C$ is justified.

We are interested in the effect of parameters like channel Singular values, power and channel perturbation on the degradation, $C_{\text {opt }}-C=C_{\text {opt }}-C_{\text {pert }}$. This requires evaluating equation 19 over a wide range of these parameters. Since equation 19 is based on the (a) approximation of $C$ by equation 16 and (b) Taylor's series approximation in equation 17 , there is a need to study the validity of these approximations over the range of variation of desued parameters. If equation 16 holds, then $\Delta \lambda_{i}(\mathbf{X})$ is a first order perturbation in $\lambda_{i}(\mathbf{X})$ and hence the higher powers of $\frac{\Delta \lambda_{i}(\mathbf{X})}{\lambda_{1}(\mathbf{X})}$ are negligible thereby validating the Taylor series approximation. Thus only the validity of equation 16 needs to be verified. Note that equation 16 holds if the (a) approximation of equation 7 by equation 8 holds (b) equation 9 holds for $\mathbf{A X}$ of equation 8 (c) $\lambda_{i}(\mathbf{A}+\mathbf{A} \mathbf{A})=\lambda_{i}(\mathbf{A})+\underline{v}_{i}^{*} \Delta \mathbf{A} \underline{v}_{i}$ holds. Effect of the parameters present in equation 19 on these approxmations are detailed next.

Effect of Perturbation Norm ( $\mid \Delta \mathbf{A} \|)$ on validity of equation 16: Clearly the first order approximation, $\lambda_{i}(\mathbf{A}+\mathbf{A A})=$ $\lambda_{i}(\mathbf{A}+\triangle \mathbf{A})+\underline{v}_{i}^{*} \triangle \mathbf{A} \underline{v}_{i}$ hinges on the fact that perturbation norm $\|\triangle \mathbf{A}\|$ is small. Hence equations 16 and 19 are valid for small perturbation norms.

Effect of Power $\mathbf{P}$ on validity of equation 16: It is clear from equation 19 that total power $\mathbf{P}$ is a parameter that can be used to combat the estimation errors which necessitates to document the effect of $\mathbf{P}$ on validity equation 16 over a wide range. Using equation 6, $\mathbf{Q}_{\text {opt }}$ can be written as $\mathbf{Q}_{\text {opt }}=\overline{\mathbf{Q}}+\frac{P}{m} \mathbf{I}_{\boldsymbol{m}}$. Since the first order validity for $\lambda_{i}(\mathbf{A}+\mathbf{A} \mathbf{A})$ is independent of $P$, equation 13 also holds and $\widehat{\mathbf{Q}}_{\text {opt }}=\overline{\widehat{\mathbf{Q}}}+\frac{P}{m} \mathbf{I}_{m}$. It is important to note that $\overline{\mathbf{Q}}$ and $\overline{\mathbf{Q}}$ are independent of $\mathbf{P}$. Hence $\mathbf{A X}=\mathbf{H}(\overline{\widehat{\mathbf{Q}}}-\overline{\mathbf{Q}}) \mathbf{H}^{*}$ is independent of $\mathbf{P}$. Also the first order approximation of eigenvalue and eigen-vector of $\mathbf{A}+\mathbf{A A}$ is independent of $\mathbf{P}$. This shows that $\mathbf{P}$ does not affect the approximations used.

$$
{ }^{1} \eta \leq \frac{\|\Delta \mathbf{A}\|}{\lambda_{m}(\mathbf{A})} \leq 0.1 \text {. Also } \lambda_{m}(\mathbf{A}+\mathbf{A A}) \geq 0.9 \lambda_{m}(\mathbf{A})
$$


Effect of Channel Singular Values on validity of equation 16

The effect of changing individual channel singular values is hard to analyze. So consider the case where all the singular values of the channel are increased by $\beta>1$. Let the new parameters be denoted with subscript $\beta$, i.e the new channel is $\mathrm{Hp}=\beta \mathrm{H}$. However, the norm of the perturbation $(\|\triangle \mathbf{H}\|)$ is same as that of the $\beta=1$ case. Though this may show that the perturbations are small with respect to the new channel, pre and post multiplication by channel matrices $\left(\mathbf{H}_{\beta}\right)$ in equation 7 necessitates to verify the first order approximations. Now $\mathbf{A p}=\beta^{2} \mathbf{A}$ and since. $P$ can be factored out, $Z_{\beta}=\beta^{-2} \mathbf{Z}$. Neglecting the second order term in $\Delta \mathbf{A}_{\beta}$, we have $\Delta \mathbf{A}_{\beta}=\mathbf{B A A}$. Since $\mathbf{A A}$ allows us to use first order approximations for eigen-values and eigenvectors of $\mathbf{A}+\mathbf{A} \mathbf{A}$, the perturbation $\triangle \mathbf{A}_{\beta}$ in $\mathbf{A}_{\beta}$ is amenable to similar approximations and residual errors. Hence we have, $\lambda_{i}\left(\mathbf{A}_{\beta}+\Delta \mathbf{A}_{\beta}\right)=\lambda_{i}\left(\mathbf{A}_{\beta}\right)+\underline{v}_{i}^{*} \Delta \mathbf{A}_{\beta} \underline{v}_{i}$. From this argument it can be seen that for $\beta \lambda_{i}(\mathbf{A}) \gg \underline{v}_{i}^{*} \triangle \mathbf{A} \underline{v}_{i}, \Delta \mathbf{Z}_{\beta}=\beta^{-3} \Delta \mathbf{Z}$. Also we have $\Delta \mathbf{V}_{\beta}=\beta^{-1} \Delta \mathbf{V}$. Using these results in equation 7 shows that the order of terms in AX does not increase with $\beta>1$. Hence the earlier approximations hold when all the channel singular values are increased.

This study shows that we can increase $P$ and channel singular values arbitrarily while using equation 19 .

\section{OBSERVATIONS}

In this section we present some observations on the variation of degradation $C_{\text {opt }}-C$ due to changes in power, channel singular values and the perturbation norm. All the results presented are for the channel used in figure 1 . Since figure 1 shews that $C_{\text {pert }}$ is a good approximation for $\mathrm{C}$, we present results for $C_{\text {opt }}-C_{\text {pert }}$.

\section{Effect of $\mathbf{A H}$}

Decreasing $\|\Delta \mathbf{H}\|$ (and hence $\|\Delta \mathbf{A}\|$ ) reduces the degradation. This is shown by equation 16 when $\|\Delta \mathbf{A}\| \rightarrow 0, \mathbf{a} ; \rightarrow 0$ thereby making $C_{\text {pert }} \rightarrow \mathrm{C}$. Equation 20 shows that the upper bound varies as the square of $\|\Delta \mathbf{A}\|$. Further, smaller the || $\triangle \mathbf{H} \|$ better is the first order approximation. These observations are shown clearly in figure 1.

\section{Effect of Total Power $P$}

Equation 19 shows clearly that increasing $P$ reduces the bound in the degradation. Further note that this decrease is approximately proportional to $P^{-2}$. Thus the degradation in capacity can be combated by increasing power. These observations are highlighted in figure 2 , for the 4 × 3 channel of figure 1. @ denotes the factor by which the power is amplified. Also, as $P \rightarrow \infty$, $C_{\text {pert }} \rightarrow C$.

\section{Effect of Channel Singular Values}

Here all the singular values of the channel are increased by the factor $\beta \geq 1$ while the perturbations ( $\mathrm{AH}$ ) are same as in the $\beta=1$ case. Choosing $P$ as in Appendix $A$, results in $P$ a $\beta^{-2}$. Then it can be seen from equation 19, with the assumption $\beta \lambda_{i}(\mathbf{A})>\mathrm{a}$; , that degradation falls approximately as $\beta^{-2}$. Note that degradation reduces despite the fact that $P$ is also reduced. Also, $\beta \mathbf{H}+\mathbf{A H} \rightarrow \beta \mathbf{H}$ for $\beta>1$. As a result, we see that the degradation reduces as the channel norm increases. These are summarized in figure 3 which shows the degradation for the channel of figure 1 for various $\beta$.
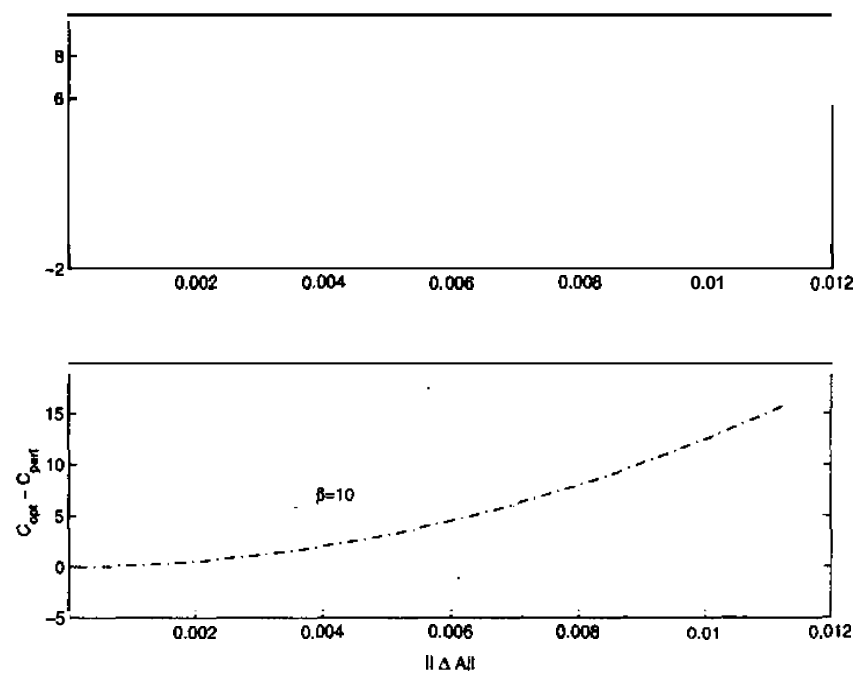

Fig. 2. Variation of capacity degradation with $\mathbf{P}$. The channel of figure 1 is used with $\beta$ indicating the power multiplication factor.
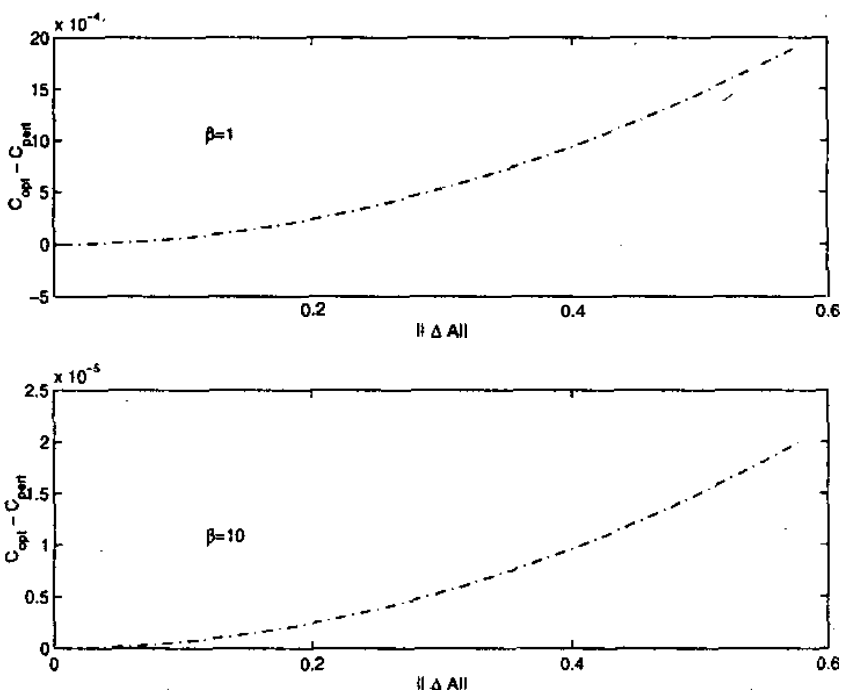

Fig. 3. Variation of capacity degradation with Channel singular valuer for channel of figure $t, \beta$ indicating the multiplication factor

\section{N. EXTENSION TO MIMO-OFDM SYSTEMS}

The earlier results were reported for frequency Bat channels. These results can be extended to any communication system where $C$ and $C_{\text {opt }}$ are obtained by expressions similar to those in equation 3. The capacity of MIMO-OFDM system over frequency selective channels using $N$ tones is [5], $C_{\text {opt }}=\frac{1}{N} \log \operatorname{det}\left[\mathrm{I}_{n N}+\right.$ $\left.\mathbf{H Q}_{\text {opt }} \mathbf{H}^{*}\right]$ and $C=\frac{1}{N} \log \operatorname{det}\left[\mathrm{I}_{n N}+\mathbf{H} \widehat{\mathbf{Q}}_{o p t} \mathrm{H}^{*}\right]$ where $\mathbf{H}$ is an $n N \times m N$ block diagonal matrix with $\mathrm{H}=\operatorname{diag}\left\{\mathbf{H}_{k}\right\}_{k=0}^{N-1}$. Since these expressions are similar to those in equation 3 , earlier results can be extended to MIMO-OF'DM systems and we present them for completeness.

The submatrices $\mathbf{H}_{\boldsymbol{k}}$ are $n \times \mathbf{m}$ matrices denoting the $k^{\text {th }} \mathrm{DFT}$ 
co-efficient of the channel, i.e if $\mathbf{H}(l)$ denotes the $n \boldsymbol{x} \boldsymbol{m}$ channel for the $l^{t h}$ path, then $\mathrm{HI}_{\mathrm{I}}=\sum_{l=1}^{L} \mathbf{H}(l) e^{-j 2 \pi \frac{k_{l}}{N}}$. We have assumed that there are $L$ distinguishable paths. We refer to $\mathrm{H}_{\mathrm{I}}$, as the channel for the $k^{\text {th }}$ tone. Also $\mathbf{Q}_{\text {opt }}$ is the $m N x m N$ block diagonal correlation matrix with $\mathbf{Q}_{\text {opt }}=\operatorname{diag}\left\{\boldsymbol{\Sigma}_{k}\right\}_{k=0}^{N-1}$ and $\boldsymbol{\Sigma}_{k}$ is the $\boldsymbol{m} \times \boldsymbol{m}$ correlation-matrix of the symbols on the $k^{\text {th }}$ tone. Similarly $\widehat{\mathbf{Q}}_{\text {opt }}=\operatorname{diag}\left\{\overline{\boldsymbol{\Sigma}}_{k}\right\}_{k=0}^{N-1}$. Further there exists a power constraint $P$ such that $\operatorname{Trace}\left(\widehat{\mathbf{Q}}_{\text {opt }}\right)=\operatorname{Trace}\left(\mathbf{Q}_{\text {opt }}\right) \leq P$. With these definitions we can write $C=\frac{1}{N} \sum_{k=0}^{N-1} \log \operatorname{det}\left[\bar{I}_{n}+\right.$ $\mathbf{H}_{k} \widehat{\mathbf{\Sigma}}_{k} \mathbf{H}_{k}^{*}$ ] which shows the decomposition into $N$ frequency flat channels. Note that the power constraint forces these channels to dependent.

The channel for each tone $\mathrm{H}_{k}$ is estimated as [ $\left.\mathbf{H} \Delta \mathbf{H}\right]_{k}$ for each tone $\mathrm{k}$ independently.. Further, $\mathrm{A}=\mathrm{H}^{*} \mathrm{H}, \mathrm{AI},=\mathbf{H}_{k}^{*} \mathbf{H}_{k}$, $[\mathbf{A}+\Delta \mathbf{A}]_{k}=[\mathrm{H}+\Delta \mathrm{H}]_{k}^{*}[\mathbf{H}+\Delta \mathbf{H}]_{k}$ and $[\mathrm{A}+\mathrm{AA}]=$ $[\mathrm{H}+\Delta \mathrm{H}]^{*}(\mathrm{H}+\mathrm{AH}]$ The norm of perturbation for each $\mathbf{A}_{k}$ is $\left\|\triangle \mathbf{A}_{k}\right\|$. Wedenoteby $\lambda_{j}\left(\mathbf{A}_{k}\right)$ the $j^{t h}$ eigen-value of $\mathbf{A}_{k}$ and for each $\mathrm{k}$, they are arranged in decreasing order.

\section{A. Perturbation Analysis}

We assume $\mathrm{H}$ to be full rank with distinct eigen-values. To highlight independent perturbations, we provide the following expressions that can be easily derived from earlier results. Let $\mathbf{D}_{(i, i)}(k)$, be the $i^{t h}$ singular value of $\mathrm{H}_{k}, \underline{v}_{i}(k), \underline{u}_{i}(k)$ be the $i^{t h}$ eigen-vector of $\mathbf{A}_{k}$ and $\mathrm{H}_{k} \mathrm{H}_{k}^{*}$ respectively, $\alpha_{(k, i)}=$ $\underline{v}_{i}(k)^{*}[\Delta \mathbf{A}]_{k} \underline{v}_{i}(k), \mu_{(k, i)}=\lambda_{i}\left(\mathbf{A}_{k}\right)+\alpha_{(k, i)}, 1 \leq i \leq \boldsymbol{m}, \boldsymbol{O} \leq$ $k \leq N-1$.The first order approximation, $C_{\text {pert }}$, of $C$ is

$$
\begin{gathered}
C_{p e r t}=\frac{1}{N} \sum_{k=0}^{N-1} \sum_{i=1}^{m} \log \left(\frac{\alpha_{(k, i)}}{\mu_{(k, i)}}\right. \\
\left.+\frac{\lambda_{i}\left(\mathbf{A}_{k}\right)}{N m}\left[P+\sum_{l=0}^{N-1} \sum_{j=1}^{m} \frac{1}{\mu_{(l, j)}}\right]\right)
\end{gathered}
$$

Further simplifications can be made by noting $\alpha_{(k, i)}=2 \mathbf{D}_{(i, i)}(\mathrm{k})$ $\operatorname{Real}\left(\underline{u}_{i}(k)^{*}[\Delta \mathbf{H}]_{k} \underline{v}_{i}(k)\right)+\left\|[\Delta \mathbf{H}]_{k} \underline{v}_{i}(k)\right\|_{2}^{2}$.

The degradation $C_{\text {opt }}-C_{\text {pert }}$ is similar to equation 19 and is given by,

$$
\begin{gathered}
C_{\text {opt }}-C_{\text {pert }}=\frac{N}{2 \ln 2}\left(\frac{m}{P+\gamma}\right)^{2} \sigma \text { with } \\
\sigma=\sum_{k=0}^{N-1} \sum_{i=1}^{m} \beta_{(k, i)}^{2}-\frac{1}{N m}\left(\sum_{k=0}^{N-1} \sum_{i=1}^{m} \beta_{(k, i)}\right)^{2} \\
\text { where } \beta_{(k, l)}=\left(\frac{\alpha_{(k, l)}}{\lambda_{l}\left(\mathbf{A}_{k}\right)\left(\lambda_{l}\left(\mathbf{A}_{k}+\alpha_{(k, l)}\right)\right.}\right)
\end{gathered}
$$

An upper bound similar to equation $\mathbf{2 0}$ can be easily derived. We see that instead of using $\|\Delta \mathbf{A}\|$ for the entire matrix, we use the perturbations $\left\|\Delta \mathbf{A}_{\boldsymbol{k}}\right\|$ for individual channels. However, we see that due to the power constraint, degradation over $\mathrm{H}_{k}$ is dependent on the perturbation on different tones. The effect of the channel singular values, power and perturbation on $C$ are as mentioned earlier.

\section{CONCLUSION}

An analysis of the variation of MIMO capacity in the presence channel perturbations has been carried out. The channel perturbations are assumed to be small so that first order perturbation analysis of MIMO capacity can be carried out. The fact that most channel estimation algorithms yield small errors motivates the aforesaid analysis. Expressions and bounds for the MIMO capacity in presence channel perturbations are derived. The validity of these expressions is also studied. It is shown that the degradation and has an approximate inverse square relation with power used and the channel singular values. The degradation decreases with the channel perturbation and the upper bound derived shows that the degradation varies as the square of the perturbation norm. These results show that degradation can be reduced by using higher power and using a channel with high singular values. All the results derived can be extended to a broader class of communication systems of which results for MIMO-OFDM systems over frequency selective channels are presented.

\section{APPENDIX A. Power Considerations}

Using the derivation in [6], for full rank channels, it can he shown that

$$
(\mathbf{1}-\eta) \lambda_{i}(\mathbf{A}) \leq \lambda_{i}(\mathbf{A}+\Delta \mathbf{A}) \leq(1+\eta) \lambda_{i}(\mathbf{A}),
$$

where $1 \leq i \leq \boldsymbol{m}$ where $\eta=\left\|\mathbf{A}^{-1}[\Delta \mathbf{A}]\right\|$. Hence $\eta \leq \frac{\|\Delta \dot{A}\|}{\lambda_{m}(\mathbf{A})}$. Since $\lambda_{\boldsymbol{i}}(\mathbf{A}+\mathrm{A} A) \geq 0, \forall i$ the lower bound in equation $\mathbf{2 3}$ becomes trivial for $\eta>\mathbf{1}$. However for perturbations which satisfy, $\|\Delta \mathbf{A}\|<\lambda_{m}(\mathbf{A})$, equation 23 shows $\lambda_{i}(\mathbf{A}+\mathrm{A} A)>0$ if $\lambda_{i}(\mathbf{A})>0$. Hence such perturbations do not reduce rank of full rank channels.

If $\lambda_{m}(\mathrm{~A}+\mathrm{AA})>0$, as is the case with perturbations that retain the rank, $P \geq \frac{m \kappa}{\lambda_{m}(\mathbf{A}+\Delta \mathbf{A})}$ for all $\kappa \geq 1$ is sufficient to prevent use of iterative water filling [1] in calculating $\lambda_{i}\left(\hat{\mathbf{Q}}_{\text {opt }}\right)$. Then choosing $\kappa=1+\eta$ and using equation 23. $P \geq \frac{m}{\lambda_{m}(\boldsymbol{A})}$, which is sufficient to to prevent use of water filling in calculating $\lambda_{\boldsymbol{i}}\left(\mathbf{Q}_{\mathbf{o p t}}\right)$. The full rank assumption of $\mathrm{A}$ is used here. The choice of $P \geq \frac{m(1+\eta)}{\lambda_{m}(\mathbf{A}+\triangle \mathbf{A})}$ helps us to avoid water filling for true and measured channels. The transmitter obtains $\lambda_{m}(\mathbf{A}+\mathbf{A A})$ and calculates $P$ for a given upper bound on $\|\triangle \mathbf{A}\|$. Even though $P$ can be further reduced, this choice is used for simplicity.

\section{REFERENCES}

[1] I. E. Telatar, "Capacity of Multi-antenna Gaussian Channels", European Transactions on Telecommunications, vol. 10, no. 6, November-December 1999.

[2] Roger A. Horn, Charles A. Johnson, "Matrix Analysis", Cambridge University Press, 1990.

[3] J. H. Wilkinson, "The Algebraic Eigenvalue Problem", Clarendon Press, Oxford, England, 1965.

[4] M. R. Bhavani Shankar, K. V. S. Hari, "On the Variations in Capacity of MIMO Communication Systems to Channel Perturbations", submitted to IEEE Transacrions on Communications.

[5] Helmut Bolcskei, D. Gesbert, A. Paulraj "On the capacity of OFDM-Based Spatial Multiplexing Systems", IEEE Transactions on Communications, vol. 50, no. 2, pp. 225-234, February 2002.

[6] Roy. Mathias, "Spectral Perturbation Bounds for Positive Definite Matrices". SIAM Journal of Matrix Analysis and Applications, vol. 18, no. 4, October 1997,pp 959-980 